\title{
Massive sequencing of artisan cheeses from raw sheep's milk
}

\begin{abstract}
Lactic acid bacteria (LAB) are used in the food industry to confer aromatic characteristics and their antibacterial capacity. In this study the native flora of LAB that participates in the traditional fermentation of semi-hard cheeses made with raw sheep's milk from the region of Andalusia, Spain was analyzed. Three samples of four different commercial cheeses were taken. Massive sequencing was carried out to identify the lactic and accompanying flora. Predominant lactic flora was Lactococcus lactis, Streptococcus thermophilus, Lactobacillus paracasei and Lactococcus raffinolactis, and to a lesser extent other species of the genera Lactobacillus, Streptococcus, Pediococcus and Leuconestoc. The accompanying flora was composed of species of the genera Mycoplasma, Pseudomonas, Acinetobacter, Chryseobacterium, Mannheimia, Trueperella, Enterococcus, Vibrio, Serratia, Macrococcus, Staphylococcus, Massilia, Flavobacterium, Yersinia, Gallaecimonas, Hafnia, Leclercia, Obesumbacterium, Morganella and Kluyvera. These results show that modern molecular techniques are very good tools to identify natural LABs of artisanal dairy products. The characterization of the native flora of the artisanal cheese allows us to evaluate the microbiological diversity of the natural population of LAB and the symbiosis with another type of flora.
\end{abstract}

Keywords: artisan cheese, metagenomic, bacteria, pasteurized milk
Volume 6 Issue 5 - 2019

\author{
Ruiz Julia,' Etcheverría Analía,' Padola Nora,' \\ Kruger Alejandra,' Medina Luis² \\ IImmunochemistry and Biotechnology Laboratory, Tandil Veterinary \\ Research Center (CIVETAN), Argentina \\ ${ }^{2}$ Department of Bromatology and Food Technology, University of \\ Córdoba, Spain
}

Correspondence: Ruiz Julia, Immunochemistry and Biotechnology Laboratory, Tandil Veterinary Research Center (CIVETAN), CONICET-UNCPBA, Faculty of Veterinary Sciences, UNCPBA,Tandil,Argentina, Email jruiz@vet.unicen.edu.ar

Received: August 22, 2019 | Published: September 24, 2019 Abbreviations: LAB, lactic acid bacteria; DNA,
Deoxyribonucleic acid

\section{Introduction}

Hundreds of cheese varieties are produced in the world based on the differences both in the type of milk used and in the method of production. However, only a small number of these varieties have commercial importance and most of them are produced and consumed locally. The production of cheese is essentially achieved by joining four ingredients: milk, rennet, microorganisms and salt. The production of lactic acid from lactose is the main biochemical change in the production of cheese, responsible for lactic acid bacteria (LAB). These starter bacteria reduce the $\mathrm{pH}$, form the dough and allow the rejection of water. The demand for cheese in the early 1900s generated large-scale production and therefore the use of pasteurized milk as a criterion of hygiene. This process involves the elimination of the natural flora of LAB contained in milk and the possible generation of a variety of flavors and aromas. Consequently, to make cheese from pasteurized milk, an external source of LAB initiators is needed.

The production of raw milk cheeses has long been practiced in Europe, particularly in Spain, France, Italy and Switzerland. Raw milk cheeses are often characterized by richer and stronger flavour intensity than cheeses made from pasteurized milk, ${ }^{1}$ and they are considered more natural. Andalusia has a great variety of excellent traditional cheeses quality and linked to the territory from which they come. Each of these cheeses is characterized by various quality attributes such as a certain race native farming, grazing-based food, as well as knowledge of the master cheese passed down generation after generation, among others. ${ }^{2}$ The current demand for fresh and easy to prepare products has brought an increase in the market of minimally processed products. This trend responds to the generalized idea that these foods are healthier. However, it must be borne in mind that it is raw food, which requires extreme good handling conditions and other techniques that allow some microbial inactivation. Minimally processed foods are fresh and, therefore, raw. This implies that the health risks may be higher than those of foods that have been treated with any technological process. Therefore, more common pathogenic microorganisms can be transmitted, among them, Listeria monocytogenes, Escherichia coli O157: H7, Salmonella, among others.

The strains that make up the microflora in raw milk and therefore in the cheese made with it have relevant functions in terms of conferring attributes of protection against pathogenic bacteria, generation of flavors and flavor or modification of texture. ${ }^{3}$ Genomic identification of promising species of $\mathrm{LAB}$ for development of novel starter culture candidates was the prime aim of the present study.

\section{Materials and methods}

\section{Cheese sampling}

Three samples of four semi-hard commercial cheeses of raw sheep's milk cheese were taken: from the center, the middle and the crust. A total of 12 samples were processing for massive sequencing.

\section{DNA extraction from cheese samples}

DNA extraction was performed with the Power Food Microbial DNA Isolation Kit (MO BIO, ref 21000-50). $1.8 \mathrm{ml}$ of microbial food culture was pelleted by centrifugation and resuspended in lysis buffer. The supernatant was transferred to a bead beating tube containing beads designed for small cell (microbial) lysis and vortex mixed using a specially designed MO BIO Vortex Adapter. After the protein and 
inhibitor removal steps, total genomic DNA was captured on the MO BIO Laboratories flat bottom silica spin column. The bound DNA was washed and eluted from the spin column membrane.

\section{Preparation of the amplicons and library}

Metagenomic kit Ion 16S (pooles primers V2-48 y V3-7, 7-9) was used (Thermo Fisher, ref A26216). This allowed the rapid and complete analysis of mixed microbial populations through the sequencing flow of Ion Torrent TM semiconductors.

The Seuser Ion Plus fragments library Kit was used, an integral component of the Personal Genome Machine TM (Thermo Fisher, ref 4471252). This kit allowed to design libraries of high-quality DNA through several workflows with an input of $100 \mathrm{ng}$ of DNA.

\section{Library enrichment and chip loading}

The PGM HiQ View Chef Ion Kit (Thermo Fisher, ref A29902) was used. The templates were prepared, the loading of chips and the sequencing of libraries of $200 \mathrm{bp}$ in the Ion Chef ${ }^{\mathrm{TM}}$ system and in the Ion $\mathrm{PGM}^{\mathrm{TM}}$ system.

Table I Information related to samples

\section{Sequencing and analysis}

We use the Ion PGM equipment, chip 318. Kit Ion PGM HiQ View Sequencing Kit (Thermo Fisher, ref A30044). The 200 and 400 base pair libraries were sequenced using the Ion OneTouch ${ }^{\mathrm{TM}} 2$ system combined with the Ion PGM ${ }^{\mathrm{TM}}$ system. The analysis of the Ion $\mathrm{PGM}^{\mathrm{TM}}$ system data was performed with the Ion Reporter ${ }^{\mathrm{TM}}$ software.

\section{Results}

Information related to samples (Table 1).

\section{Sequence analysis with Ion Reporter}

Ion Reporter uses the QIIME open bioinformatics source to produce diversity analyzes and visualizations. The results of alpha diversity describe the diversity within a single sample at the Species, Gender and Family levels. The beta diversity results describe the diversity among multiple samples at the species, genus and family level (Table 2).

\begin{tabular}{|c|c|c|c|c|c|}
\hline Barcode Name & Sample & Bases & $\geq \mathbf{Q 2 0}$ & Reads & $\begin{array}{l}\text { Mean Read } \\
\text { Length }\end{array}$ \\
\hline NO barcode & none & $4,21,54,975$ & $3,85, I I, 027$ & $2,02,922$ & $208 \mathrm{bp}$ \\
\hline lonXpress_00I & $\mathrm{Al}$ & $4,65,80,044$ & $4,45,06,4 \mid 3$ & $1,85,693$ & $251 \mathrm{bp}$ \\
\hline IonXpress_002 & A2 & $5,92,05,232$ & $5,66,35,88$ I & $2,35,520$ & $249 \mathrm{bp}$ \\
\hline lonXpress_003 & A3 & $12,10,97,845$ & $11,59,23,675$ & $4,86,327$ & $250 \mathrm{bp}$ \\
\hline IonXpress_004 & $\mathrm{BI}$ & $4,80,13,228$ & $4,60,23,378$ & I,9I,976 & $252 \mathrm{bp}$ \\
\hline IonXpress_005 & B2 & $8,74,19,483$ & $8,36,46,502$ & $3,47,482$ & $247 \mathrm{bp}$ \\
\hline IonXpress_006 & B3 & $5,66,69,596$ & $5,40,91,466$ & $2,28,998$ & $250 \mathrm{bp}$ \\
\hline IonXpress_007 & $\mathrm{Cl}$ & $7,32,73,652$ & $7,00,92,992$ & $2,92,978$ & $247 \mathrm{bp}$ \\
\hline IonXpress_008 & $\mathrm{C} 2$ & $8,|6,73,88|$ & $7,79,72,444$ & $3,30,729$ & $251 \mathrm{bp}$ \\
\hline IonXpress_009 & $\mathrm{C} 3$ & $6,76,27,167$ & $6,45,90,193$ & $2,68,960$ & $24 I \mathrm{bp}$ \\
\hline IonXpress_00I0 & DI & $8,72,95,056$ & $8,29,84,652$ & $3,61,688$ & $541 \mathrm{bp}$ \\
\hline IonXpress_00II & D2 & $7,49,10,425$ & $7, \mid 14,86,030$ & $3,07,600$ & $244 \mathrm{bp}$ \\
\hline IonXpress_0012 & D3 & $4,08,03,|15|$ & $3,89,42,102$ & $1,69,602$ & $241 \mathrm{bp}$ \\
\hline
\end{tabular}

Table 2 Predominant flora in cheese samples

\begin{tabular}{llll}
\hline Sample & Flora & Center (\%) & Meddle (\%) \\
\hline Cheese A & Chryseobacterium haifense & $<0.05$ & $<0.05$ \\
& Lactobacillus casei & $<0.05$ & $<0.05$ \\
& Lactobacillus paracasei & 0.13 & 0.57 \\
& Lactococcus lactis & 7.76 & 4.61 \\
& Lactococcus raffinolactis & 0.47 & 0.55 \\
Streptococcus thermophilus & 32.08 & 29.37 \\
Acinetobacter ursingii & $<0.05$ & $<0.05$ \\
& Pseudomona vranovensis & 0.2 & $<0.05$ \\
Mycoplasma bovis & $<0.05$ & $<0.05$
\end{tabular}


Table Continued...

\begin{tabular}{|c|c|c|c|c|}
\hline \multirow{16}{*}{ Cheese B } & Trueparella pyogenes & $<0.05$ & $<0.05$ & $<0.05$ \\
\hline & Corynobacterium & $<0.05$ & $<0.05$ & $<0.05$ \\
\hline & Macrococcus caseolyticus & $<0.05$ & $<0.05$ & $<0.05$ \\
\hline & Enterococcus faecalis & 0.13 & 0.15 & 0.17 \\
\hline & Lactobacillus & 0.07 & 0.11 & 0.12 \\
\hline & Lactobacillus brevi & $<0.05$ & $<0.05$ & $<0.05$ \\
\hline & Lactobacillus paracasei & 3.03 & 3.27 & 2.13 \\
\hline & Lactobacillus sp. & $<0.05$ & 0.06 & $<0.05$ \\
\hline & Lactococcus & 0.16 & 0.17 & 0.21 \\
\hline & Lactococcus lactis & 6.69 & 6.89 & 9.7 \\
\hline & Lactococcus raffinolactis & 0.15 & 0.34 & 0.52 \\
\hline & Streptococcus & 0.13 & 0.16 & 0.21 \\
\hline & Streptococcus salivarius & $<0.05$ & $<0.05$ & $<0.05$ \\
\hline & Streptococcus thermophilus & 32.19 & 35.1 & 27.1 \\
\hline & Streptococcus uberis & $<0.05$ & $<0.05$ & $<0.05$ \\
\hline & Serratia & $<0.05$ & $<0.05$ & $<0.05$ \\
\hline \multirow[t]{16}{*}{ Cheese C } & Lactobacillus & 0.15 & 0.31 & 0.08 \\
\hline & Lactobacillus diolivorans & 0.31 & 0.13 & 0.38 \\
\hline & Lactobacillus paracasei & 2.97 & 2.42 & 0.69 \\
\hline & Lactobacillus sp. & $<0.05$ & $<0.05$ & $<0.05$ \\
\hline & Lactobacillus zeae & 0.13 & $<0.05$ & 0.11 \\
\hline & Pediococcus & $<0.05$ & $<0.05$ & $<0.05$ \\
\hline & Pediococcus pentosaceus & 0.12 & 0.08 & $<0.05$ \\
\hline & Lactococcus & 0.11 & 0.14 & 0.13 \\
\hline & Lactococcus lactis & 4.13 & 3.85 & 2.4 \\
\hline & Lactococcus raffinolactis & 0.27 & 0.24 & 0.26 \\
\hline & Streptococcus & 0.12 & 0.18 & 0.12 \\
\hline & Streptococcus thermophilus & 40.52 & 39.29 & 46.31 \\
\hline & Streptococcus uberis & $<0.05$ & $<0.05$ & $<0.05$ \\
\hline & Manheimia varigena & $<0.05$ & $<0.05$ & $<0.05$ \\
\hline & Mycoplasma agalactiae & $<0.05$ & $<0.05$ & $<0.05$ \\
\hline & Mycoplasma bovis & 1.04 & 0.81 & 0.81 \\
\hline \multirow[t]{11}{*}{ Cheese D } & Enterococcus & $<0.05$ & $<0.05$ & $<0.05$ \\
\hline & Lactobacillus & $<0.05$ & $<0.05$ & $<0.05$ \\
\hline & Lactobacillus futsaii & $<0.05$ & $<0.05$ & $<0.05$ \\
\hline & Lactobacillus paracasei & 0.09 & 0.16 & 0.22 \\
\hline & Lactobacillus sakei & $<0.05$ & $<0.05$ & $<0.05$ \\
\hline & Leuconestoc & $<0.05$ & 0.06 & 0.01 \\
\hline & Leuconestoc mesenteroides & 0.58 & 0.38 & 0.82 \\
\hline & Leuconestoc pseudomesenteroides & $<0.05$ & 0.08 & 0.08 \\
\hline & Lactococcus & $<0.05$ & 0.22 & 0.11 \\
\hline & Lactococcus lactis & 6.73 & 8.08 & 4.22 \\
\hline & Lactococccus raffinolactis & 19.38 & 21.58 & 16.19 \\
\hline
\end{tabular}


Table Continued...

\begin{tabular}{llll} 
Lactococcus sp. & 0.14 & 0.15 & 0.09 \\
Streptococcus parauberis & 0.06 & 0.11 & $<0.05$ \\
Hafnia alvei & $<0.05$ & $<0.05$ & $<0.05$ \\
Hafnia paralvei & 0.81 & 0.79 & 1.1 \\
Kluyvera & $<0.05$ & $<0.05$ & $<0.05$ \\
Obesumbacterium proteus & 1.73 & 1.64 & 2.17 \\
Providencia & $<0.05$ & $<0.05$ & $<0.05$ \\
Serratia glossinae & 0.27 & 0.21 & 0.14 \\
Serratia grimesii & 0.6 & 0.33 & 0.76 \\
Serratia liquefaciens & 0.17 & 0.14 & 0.13 \\
Serratia quinovorans & 0.56 & 0.31 & 0.61 \\
Yersinia intermedia & $<0.05$ & $<0.05$ & $<0.05$ \\
Pseudomonas & 0.21 & 0.38 & 0.66 \\
Gallaecimonas xiamennensis & $<0.05$ & $<0.05$ & 0.06 \\
\hline
\end{tabular}

\section{Discussion}

Artisan cheeses are characterized by a rich source of various LAB with interesting functional properties. In recent years, many researchers have focused on the isolation of native LAB from artisanal cheeses made with raw milk without the addition of starters cultures. As in this study, other researchers have isolated LAB from cheeses made with raw sheep's milk. AvnI Kirmac1 ${ }^{4}$ in raw sheep milk cheeses from Turkey, Ramírez-López ${ }^{5}$ in raw goat milk cheeses in Mexico, Vernile et al., ${ }^{6}$ in the classic Pecorinos cheese from Italy, among others. The preliminary identification of isolates from dairy products generally shows the presence of four dominant LAB genera: Lactococcus spp., Enterococcus spp., Leuconostoc spp., Lactobacillus spp. and Streptococcus spp. Terzic-Vidojevic et al. ${ }^{7}$ In another study, the percentage distribution of isolated LABs from highest to lowest has been: Enterococcus spp., Lactococcus spp., Lactobacillus spp., Streptococcus spp. and Leuconostoc spp. ${ }^{4}$

These strains generally play an important role in the development of cheese flavor. Specifically, the Enterococci play an important role in the development of the typical flavor of the cheese and could be good components of cheese starter cultures. ${ }^{8}$ Identification at the species level is an important issue since it can help verify the presence of multiple isolates and distinguish groups of strains or unique strains with peculiar technological properties.

Taxonomic identification often cannot determine up to species level. The importance of knowing what type of bacteria colonizes a given environment provides information to be able to associate microbial activities with certain populations. For this reason, in this study, massive sequencing was used as a metagenomic method. ${ }^{9} \mathrm{~A}$ broad panorama of the constituent flora of the cheese was obtained. Lactococcus lactis, Streptococcus thermophilus, Lactobacillus paracasei and Lactococcus raffinolactis were detected as dominant lactic flora, and to a lesser extent (0.01-0.99) Lactobacillus brevis, Lactobacillus casei, Lactobacillus diolivorans, Lactobacillus futsaii, Lactobacillus paracasei, Lactobacillus sakei, Lactobacillus sp., Lactobacillus zeae, Lactococcus, Lactococcus raffinolactis, Lactococcus sp., Streptococcus parauberis, Streptococcus salivarius, Streptococcus uberis, Pediococcus pentosaceus,
Leuconestoc mesenteroides and Leuconestoc pseudomesenteroides. The accompanying flora was composed of species of the genera Mycoplasma, Pseudomonas, Acinetobacter, Chryseobacterium, Mannheimia, Trueperella, Enterococcus, Vibrio, Serratia, Macrococcus, Staphylococcus, Massilia, Flavobacterium, Yersinia, Gallaecimonas, Hafnia, Leclercia, Obesumbacterium, Morganella and Kluyvera. In a similar metagenomic study performed by López ${ }^{10}$ the genera Lactobacillus, Weissella and Leuconostoc were identified as dominant and more than 500 non-dominant genera.

With this information it is possible to determine the symbiotic relationships between the communities that participate in the cheese fermentation and maturation process. The use of a metagenomic analysis allowed to perform a taxonomic and functional characterization of the microbiome at a highly detailed level. This would explain the sensory and safety characteristics of semi-hard cheeses of raw sheep's milk. In this context, it is important to emphasize that the native LAB microflora that characterizes artisan cheeses generally consists of microorganisms adapted to the product, to specific environmental conditions and production technology. Therefore, the availability of valid molecular methods for a reliable allocation of species and a specific differentiation at the strain level is of great importance. ${ }^{11-13}$

\section{Conclusion}

The isolation and characterization of LAB strains from artisanal products is of great importance since it allows to increase the knowledge about the potential and the application of native strains to be used as starter cultures. The improvement and optimization of the control of the fermentation processes, allows the development of products with defined and constant properties but with characteristics such as artisan products.

\section{Acknowledgments}

None.

\section{Conflicts of interest}

The author declares there are no conflicts of interest. 


\section{Funding details}

None.

\section{References}

1. Buchin S, Delague V, Duboz G, et al. Influence of pasteurization and fat composition of milk on the volatile compounds and flavor characteristics of a semi-hard cheese. Journal of Dairy Science. 1998;81(12):3097-3108.

2. Ghahremani E, Mardani M, Rezapour S. Phenotypic and genotypic characterization of lactic acid bacteria from traditional cheese in khorramabad city of iran with probiotic potential. Applied biochemistry and biotechnology. 2015;175(5):2516-2527.

3. Speranza B, Bevilacqua A, Corbo MR, et al. Selection of autochthonous strains as promising starter cultures for Fior di Latte, a traditional cheese of southern Italy. Journal of the Science of Food and Agriculture. 2015;95(1):88-97.

4. Avnİ Kırmacı H, Özer BH, Akçelik M, et al. Identification and characterisation of lactic acid bacteria isolated from traditional Urfa cheese. International Journal of Dairy Technology. 2016;69(2):301-307.

5. Ramírez-López C, Vélez-Ruiz JF. Isolation, Characterization and Selection of Native Lactic Bacteria of Milk and Fresh Goat Cheese. Technological information. 2016;27(6):115-128.

6. Vernile A, Giammanco G, Spano G, et al. Genotypic characterization of lactic acid bacteria isolated from traditional Pecorino Siciliano cheese. Dairy Science and Technology. 2008;88(6):619-629.
7. Terzic-Vidojevic A, Mihajlovic S, Uzelac G, et al. Characterization of lactic acid bacteria isolated from artisanal Travnik young cheeses, sweet creams and sweet kajmaks over four seasons. Food microbiology. 2014;39:27-38.

8. Awad S, Ahmed N, El Soda M. Evaluation of isolated starter lactic acid bacteria in Ras cheese ripening and flavour development. Food Chemistry. 2007;104(3):1192-1199.

9. Ávila RO, Guzmán GH. Construction of a metagenomic library of symbiont bacteria from the mantis insect intestine. Young people in science. 2015;1(1):126-133.

10. López EB. Between opportunities and obstacles. What reveals the process of insertion of artisan Cotija cheese in the formal economy. University Journal of Social Sciences and Humanities. 2016;(7):84-111.

11. Giraffa G. Enterococci from foods. FEMS Microbiology Reviews. 2002;26(2):163-171.

12. Riesenfeld CS, Schloss PD, Handelsman J. Metagenomics: genomic analysis of microbial communities. Annu Rev Genet. 2004;38:525-552.

13. Ruiz MJ, Colello R, Padola NL, et al. Inhibitory effect of Lactobacillus spp. on bacteria involved in foodborne diseases. Argentine Journal of Microbiology. 2017;49(2):174-177. 